\title{
RELATO DE CASO: DEFICIÊNCIA DE TIAMINA COMO CAUSA DE DESCOMPENSAÇÃO CARDÍACA
}

\author{
Valdeon Caetano Rodrigues JUNIOR, Ana Lúcia Moulin Moreira de CARVALHO*, Grazziela Vieira \\ CIRQUEIRA, Arianne Alves COSTA, Nadia Arenas VERSALI, Bernardo Borges MARQUES, Flávia de \\ Almeida MIGUE \& Fernanda Nobre TORRES
}

Hospital Universitário São José. Belo Horizonte, Minas Gerais, Brasil.

*Autor para correspondência: analuciamoulin@yahoo.com.br

DOI: http://dx.doi.org/10.18571/acbm.148

\section{RESUMO}

O beribéri é uma doença causada pela falta de vitamina B1 (tiamina) no organismo, decorrente de várias causas, que pode gerar alterações nervosas, cerebrais e cardíacas. Objetivo: relatar o caso de uma paciente com insuficiência cardíaca, que obteve regressão dos sinais e sintomas após medicação com tiamina, corroborando na literatura descrita sobre o tema, pela raridade do caso. Metodologia: relato de caso. Resultados: a administração de tiamina por via parenteral, resultou em melhora parcial do quadro clínico após 3 dias e melhora total do quadro 5 dias após o uso de tiamina, comprovada por novo ecocardiograma. Conclusão: manifestações cardiovasculares (beribéri úmido) da deficiência de tiamina são raras e sua forma extrema, de Shoshin, é rapidamente fatal a menos que a terapia específica seja dada. $O$ tratamento consiste na administração de tiamina, com melhora clínica rápida após sua suplementação.

Palavras chave: Beribéri; Deficiência de tiamina; Insuficiência cardíaca.

\begin{abstract}
Beriberi is a disease caused by a lack of vitamin B1 (thiamine) in the body, due to several causes, which can lead to nervous, brain and heart changes. Objective: to report the case of a patient with heart failure, who obtained regression of the signs and symptoms after thiamine medication, corroborating in the literature described on the subject, the rarity of the case. Methodology: case report. Results: administration of thiamine parenterally resulted in partial improvement of the clinical picture after 3 days and total improvement of the picture 5 days after the use of thiamine, confirmed by a new echocardiogram. Conclusion: cardiovascular (wet beriberi) manifestations of thiamine deficiency are rare and its extreme form, Shoshin's, is rapidly fatal unless specific therapy is given. Treatment consists of the administration of thiamine, with rapid clinical improvement after its supplementation.
\end{abstract}

Keywords: Beriberi; Thiamine deficiency; Heart faluire.

\section{Introdução}

O beribéri é uma doença provocada pela falta de vitamina B1 (tiamina) no organismo, caracterizado por alterações nervosas, cerebrais e cardíacas. A deficiência de vitamina B1 pode ocorrer por diversas causas, entre elas a baixa absorção pelo intestino, diarréias prolongadas, vômitos, pacientes em uso de altas doses de diuréticos de alça, etilistas, em diálise peritoneal ou hemodiálise, em pacientes com nutrição parenteral e naqueles com tireotoxicose. Neste caso, relatamos uma paciente com insuficiência cardíaca (IC) que apresentou regressão dos sinais e sintomas após reposição de tiamina. 


\section{Biomedica Brasiliensia}

\section{Materiais e Métodos}

Foi realizada pesquisa bibliográfica através da seleção de autores para fundamentação e desenvolvimento do estudo. O relato de caso a ser descrito apresenta-se no campo da Saúde, na área de Cardiologia. A metodologia refere-se ao relato de caso, cuja paciente estudada é do sexo feminino, com 17 anos de idade, admitida no Hospital Universitário São José (HUSJ), com insuficiência cardíaca (IC) e que apresentou regressão dos sinais e sintomas após reposição de tiamina.

Os dados coletados na pesquisa através da observação/anamnese e exames complementares foram significativos para elucidar uma melhor compreensão do fenômeno pesquisado, bem como para elaborar os resultados dos dados coletados. Este relato de caso foi autorizado pela família da paciente para publicação, uma vez que a identificação da mesma fosse preservada, seguindo os preceitos éticos da resolução 466/12 do Conselho Nacional de Saúde.

\section{Relato de Caso}

TSO, 17 anos, sexo feminino, natural de Manhuaçu, portadora de estenose mitral reumática moderada, diagnosticada há 4 anos. Evoluiu com piora progressiva nos últimos meses, procurando atendimento no hospital da cidade de origem no dia 04/07/12 com história de tosse intensa com expectoração amarelada associada à dispneia em repouso e edema bimaleolar há 21 dias. Negava dispneia paroxística noturna (DPN), ortopnéia e outros sintomas. Permaneceu internada por 15 dias com diagnóstico de pneumonia comunitária e insuficiência cardíaca descompensada. Recebeu tratamento com Claritromicina durante 10 dias; digoxina $0,25 \mathrm{mg} /$ dia, micronebulização com berotec e atrovent, furosemida $80 \mathrm{mg} /$ dia e espironolactona $25 \mathrm{mg} / \mathrm{dia}$.

Durante a internação foi realizado ecocardiograma transtorácico (ECOTT), evidenciando presença de estenose mitral grave (área valvar: $0,9 \mathrm{~cm}^{2}$; gradientes máximo de 43 e médio de 26 mmHg; Escore de Block 6), insuficiência mitral leve, insuficiência tricúspide moderada, espessamento de valva aórtica, fração de ejeção do ventrículo esquerdo (FEVE) preservada, dilatação importante de ventrículo (VD) e átrio esquerdo (AE); pressão sistólica de artéria pulmonar (PSAP) de 100 mmHg. Foi encaminhada para o Hospital Universitário São José (HUSJ) no dia 19/07/2012 com proposta de abordagem cirúrgica de estenose mitral.

À admissão encontrava-se corada, hidratada no limiar, acianótica e anictérica. Alerta e consciente. Bradicárdica, hipotensa e eupneica (FC:48 bpm, PA:100/40 mmHg e FR:18 irpm). Aparelho respiratório com presença de crepitações grosseiras difusas e sibilos difusos. Aparelho cardiovascular com ritmo cardíaco regular em dois tempos, B1 hiperfonética e B2 hiperfonética, presença de sopro holosistólico $(2+/ 4+)$ em foco aórtico e sopro holodiastólico $(2+/ 4+)$ em foco mitral. Ausência de ingurgitamento jugular e refluxo hepato-jugular. Abdome plano, indolor à palpação, com ausência de massas ou visceromegalias. Edema (1+/4+) em membros inferiores, panturrilhas livres.

Após discussão da equipe do HUSJ, optou-se por tratamento percutâneo da válvula mitral com cateter-balão de Inoue, uma vez que suas características eram favoráveis (Escore de Block = $6)$.

Na enfermaria evoluiu com lipotímia, queda do estado geral, náuseas, vômitos e febre, sendo iniciado antibioticoterapia em 20/07/2012, apresentando boa resposta clínica.

Enquanto paciente aguardava valvoplastia percutânea, apresentou no dia 05/08/2012, dispnéia súbita importante, associado a escarros hemoptóicos, refratária às medidas não invasivas, sendo necessário intubação orotraqueal e internação no CTI para suporte hemodinâmico e ventilatório.

No dia 07/08/2012, foi encaminhada ao laboratório de hemodinâmica ainda em ventilação mecânica e instável hemodinamicamente para valvuloplastia percutânea. Após o procedimento, 
durante ventriculografia contrastada, identificou-se grave disfunção ventricular esquerda com hipocinesia difusa grave (FEVE 35\%), sem sinais de piora angiográfica do refluxo mitral.

Após o procedimento, foi realizado ECOTT em 07/08/2012 que evidenciou: Aumento importante de AE (47 mm); Função sistólica global do ventrículo esquerdo deprimida em grau moderado (FEVE de aproximadamente 35\%); Cardiopatia reumática: valva mitral de aspecto reumático com folheto anterior espessado abrindo-se em dome, folheto posterior fixo, fluxo turbulento a esse nível e velocidade aumentada gerando gradiente de pico e médio de respectivamente $17 \mathrm{mmhg}$ e $6,6 \mathrm{mmhg}$; Presença de regurgitação mitral moderada a importante (área do jato regurgitante: 9,46cm2); Presença de regurgitação tricúspide importante; PSAP: 70mmhg; Aumento moderado do ventrículo direito e leve do átrio direito.

Diante da suspeita clínica de Beriberi cardíaco fulminante (de Shoshin) foi iniciado tiamina $900 \mathrm{mg} /$ dia por via parenteral no dia 08/08/2012, apresentando melhora do quadro após três dias, sendo extubada e retirado drogas vasoativas. Observado melhora total dos sinais e sintomas cinco dias após uso de tiamina, foi reduzido a dose gradativamente até o dia 19/08/2012, quando o medicamento foi suspenso.

Realizado novo ECOTT dia 22/08/2012 que evidenciou: *FEVE=67\%, PSAP=66, AE=45 $\mathrm{mm}$, Cardiopatia reumática, aumento importante de AE e moderado de AD; VE com função preservada; VD hipertrófico com aumento moderado e hipocinesia leve; dupla lesão mitral com estenose leve-moderada e regurgitação moderada (valva com espessamento leve sugestivo de processo reumático, área valvar $=1,6 \mathrm{~cm}^{2}$, gradiente transvalvar médio de $06 \mathrm{mmHg}$ e máximo de $14 \mathrm{mmHg}$ ); Regurgitação tricúspide importante. Hipertensão pulmonar moderada.

No dia 23/08/2012 a paciente recebeu alta assintomática, orientada a fazer controle ambulatorial.

\section{Resultados}

Deficiência de tiamina como causa de descompensação cardíaca é raro e sua forma extrema, de Shoshin, é rapidamente fatal a menos que a terapia específica seja dada. O tratamento consiste na administração de tiamina, com melhora clínica rápida após sua suplementação.

Como o método diagnóstico, o biomarcador de atividade da transquetolase eritrocítica, é um exame caro e pouco disponível, e por não haver outro fator que explicasse as manifestações clínicas e a evolução do caso descrito e ainda considerando a gravidade do mesmo, optamos pelo teste terapêutico. A paciente apresentava história prévia de vômito, febre e uso de diurético de alça, o que justificaria a deficiência de tiamina. O beribéri era hipótese mais provável para a nossa paciente.

No caso relatado, foi evidenciado melhora total dos sinais e sintomas cinco dias após uso de tiamina via parenteral, demonstrando eficiência nos métodos adotados pela equipe.

A dose exata para o tratamento do Beribéri não está determinada, nossa paciente apresentou melhora total do quadro, inclusive com reversão da disfunção ventricular comprovada por ecocardiograma, com 900mg diárias de tiamina após cinco dias e redução gradativa da dose até sua suspensão total após 9 dias de tratamento. A paciente foi encaminhada para posterior seguimento ambulatorial.

\section{Discussão}

A tiamina é uma vitamina solúvel, essencial no metabolismo dos carboidratos. Suas principais fontes são: cereais, grãos, carnes (especialmente de porco), vegetais e laticínios1. O beribéri ou deficiência de tiamina (vitamina B1) pode ter efeitos neurológicos e cardíacos significativos1, sua depleção total corporal ocorre em aproximadamente três semanas sem 
suplementação3. Alto consumo de carboidratos, situações de estresse, como febre e infecções, além de uso prolongado de diuréticos de alça, podem agravar o quadro (PARK, et al, 2007).

Embora não se disponha de qualquer estudo controlado que demonstre o efeito favorável dos diuréticos na mortalidade da IC, seu uso para a melhora dos sintomas congestivos está bem estabelecido há décadas. Os diuréticos aumentam a natriurese e a diurese, reduzem o volume intravascular, o volume ventricular, a pré-carga, a congestão visceral e os consequentes sintomas da IC. O uso crônico e prolongado de diuréticos, tão fundamentais nos quadros de tratamento de IC, pode acarretar a deficiência da tiamina. A tiamina é uma vitamina hidrossolúvel do complexo $\mathrm{B}$, essencial para a conversão dos carboidratos e importante para o funcionamento do sistema nervoso, dos músculos e do coração. Absorvida principalmente na parte superior do duodeno, a tiamina, por meio da mucosa intestinal, é transportada para o fígado, pela circulação portal, retornando ao lúmen intestinal com a bile. Já a sua excreção é essencialmente pela urina. Pouca tiamina é excretada na bile (SHILS, et al, 2003).

A deficiência de tiamina secundária ao uso prolongado de diuréticos é bem conhecida desde o final da década de 70 (YUI, et al, 1980). A reposição de tiamina nesses cardiopatas pode resultar em aumento na fração de ejeção. No Brasil, Cunha e col. analisaram 30 cardiopatas e igual número de controles sadios. A tiamina foi analisada pelas medidas da atividade da transcetolase eritrocitária e do efeito do pirofosfato de tiamina e se observou deficiência de tiamina em $10 \%$ dos controles e em 33\% dos cardiopatas $(\mathrm{p}=0,02)$. A deficiência de tiamina foi mais frequente nos cardiopatas, ocorrendo em um terço deles, e levantando a possibilidade de que a reposição sistemática desse micronutriente possa contribuir para o melhor desempenho miocárdico nos pacientes em uso de diuréticos por período prolongado (DA CUNHA, et al, 2002).

Podemos observar quatro formas fundamentais de deficiência de tiamina: a encefalopatia de Wernicke, o beribéri seco (em que predomina o quadro de polineuropatia periférica), o beribéri úmido, no qual estão presentes os sintomas e os sinais de insuficiência cardíaca de alto débito e o shoshin beribéri (sho=dano agudo, shin=coração) associado ao choque (PEREIRA, et al, 1985).

Em relação ao diagnóstico, a atividade da transquetolase eritrocítica é o biomarcador da deficiência de tiamina. Entretanto, esse é um exame caro e não disponível na maioria dos serviços (GABRIELLI e CARUSO, 2001). Por essa razão, o teste terapêutico torna-se a forma corriqueira de confirmar os casos suspeitos (PARK, et al, 2007).

O tratamento do beribéri consiste em suporte cardiovascular e reposição de tiamina (SMITHLINE, 2007). A dose exata para o tratamento do Beribéri não está determinada, nossa paciente apresentou melhora total do quadro com 900mg diárias de tiamina após três dias, com redução gradativa da dose até sua suspensão total após 9 dias de tratamento.

Durante a investigação, não houve outro fator que explicasse as manifestações clínicas e a evolução. A paciente apresentava história prévia de vômito, febre e uso de diurético de alça, o que justificaria a deficiência de tiamina. Adicionalmente, houve resposta clínica imediata com a reposição de tiamina. Assim, apesar de não termos feito a dosagem da atividade da transquetolase eritrocítica, o beribéri tornou-se a hipótese mais provável para nosso paciente (COELHO, et al, 2007).

\section{Conclusão}

Manifestações cardiovasculares (beribéri úmido) da deficiência de tiamina são caracterizados por vasodilatação periférica com aumento do débito cardíaco, lesão miocárdica, retenção de sódio e de água e IC biventricular. Sua forma extrema, de Shoshin, é rapidamente fatal a menos que a terapia específica seja dada. O tratamento consiste na administração de tiamina, com melhora clínica rápida após sua suplementação. 


\section{Referências}

COELHO, Liana S.; HUEB, João C.; MINICUCCI, Marcos F.; AZEVEDO, Paula S.; PAIVA, Sergio A. R.; ZORNOFF, Leonardo A. M. Deficiência de tiamina como causa de cor pulmonale reversível. Arq. Bras Cardiol. 2007; vol 91 (1): e7 - e9

DA CUNHA S, ALBANESI FM F ${ }^{\circ}$, BASTOS VLFC, ANTELO DS et al. Thiamin, Selenium and Copper levels in patientes with idiopathic dilated cardiomyopathy taking diuretics. Arq. Bras Cardiol. 2002; vol 79 (n5): 460-5

GABRIELLI A, CARUSO, L. Early recognition of acute cardiovascular beriberi by interpretation of hemodynamics. J Clin Anest. 2001; 13: 230-8.

PARK JH, LEE JH, JEONG JO, SEONG IW, CHOI SW. Thiamine deficiency as a rare cause of reversible severe pulmonary hypertension. Int J Cardiol. 2007 Sep 14;121(1):e1-3. Epub 2007 Mar 7.

PEREIRA VG, MASUDA Z, KATZ A, TRONCHINI VJ. Emergência de shoshin beribéri no Brasil: aspectos clínicos, hemodinâmicos e terapêuticos. Rev Ass Méd Brasil 1985; 31: 17-9.

SHILS M, OLSON J, SHIKE M et al. Tratado de Nutrição Moderna na Saúde e na Doença 2003; 21:407-15.

SMITHLINE, HA. Thiamine for the treatment of acute decompensated heart failure. Am $\mathrm{J}$ Emerg Med. 2007; 25: 124-6.

YUI Y, ITOKAUA Y, KAWAI C. Furosemide - induced thiamine deficiency. Cardiovasc Res $1980 ; 14: 537-40$. 